Research Paper

\title{
Serum VEGF levels in the early diagnosis and severity assessment of non-small cell lung cancer
}

\author{
Yanzhen Lai ${ }^{1,3^{*}}$, Xueping Wang1,2*, Tao Zeng1,2, Shan Xing1,2, Shuqin Dai ${ }^{1,2}$, Junye Wang 1,5 , Shulin Chen ${ }^{1,2}$, \\ Xiaohui Li1,2, Ying Xie ${ }^{4}$, Yuanying Zhu1,2, Wanli Liu' ${ }^{1,2}$ \\ 1. State Key Laboratory of Oncology in Southern China, Collaborative Innovation Center for Cancer Medicine, Guangzhou, China \\ 2. Department of Clinical Laboratory, Sun Yat-sen University Cancer Center, Guangzhou, China \\ 3. Department of Experimental Research, Sun Yat-sen University Cancer Center, Guangzhou, China \\ 4. Department of Laboratory Medicine, The First Affiliated Hospital of Sun Yat-Sen University, Guangzhou, China. \\ 5. Department of thoracic surgery, Sun Yat-sen University Cancer Center, Guangzhou, China \\ * Equal contributors \\ $\triangle$ Corresponding author: liuwl@sysucc.org.cn; Department of Clinical Laboratory, Sun Yat-sen University Cancer Center, 651 Dongfeng Road East, Guangzhou \\ 510060, China. Tel./Fax: +86-20-8734-3192 \\ (c) Ivyspring International Publisher. This is an open access article distributed under the terms of the Creative Commons Attribution (CC BY-NC) license \\ (https://creativecommons.org/licenses/by-nc/4.0/). See http://ivyspring.com/terms for full terms and conditions.
}

Received: 2017.11.21; Accepted: 2018.01.21; Published: 2018.04.06

\begin{abstract}
Background: Effective biomarkers are essential to the differential diagnosis and severity assessment of non-small cell lung cancer (NSCLC). This study explored the use of the serum vascular endothelial growth factor (VEGF) levels as a biomarker with the aim of achieving better management of NSCLC.

Methods: Serum VEGF levels were assayed via enzyme-linked immunosorbent assay in 180 patients with NSCLC, 136 patients with benign pulmonary nodules, and 119 healthy controls. We additionally detected the serum concentration of three traditional biomarkers-carcinoembryonic antigen (CEA), cancer antigen (CA)-125, and cytokeratin 19 fragments (Cyfra 21-1) — to comparatively evaluate the efficiency and diagnostic value of VEGF in patients with NSCLC. We further evaluated the relationship between serum VEGF levels and clinicopathologic parameters. VEGF levels were compared between pro- and post-surgical patients using the Wilcoxon matched-pairs signed-rank test. DNA was isolated from the primary tumors. EGFR mutations were detected by Scorpions amplification refractory mutation system (ARMS).

Results: Patients with NSCLC had significantly higher serum concentration of VEGF, compared to those with benign pulmonary nodules and healthy controls $(\mathrm{P}<0.0001)$. As a diagnostic biomarker of NSCLC, VEGF had area under the curve values of 0.824 and 0.839 , sensitivities of $75.0 \%$ and $75.0 \%$, and specificities of $93.3 \%$ and $95.6 \%$ when compared with healthy people and patients with benign pulmonary nodules, respectively; notably, these values were greater than those of CA125, Cyfra 21-1 and CEA. Furthermore, a model in which VEGF was combined with CEA, CA125, and Cyfra 21-1 was more effective for NSCLC diagnosis than VEGF alone (sensitivity, $85.0 \%$ and 84.4 ; specificity, $90.0 \%$ and $91.9 \%$ vs. healthy controls and patients with benign pulmonary nodules, respectively). When use to identify early-stage NSCLC, VEGF showed a better diagnostic efficacy than other biomarkers. The pro-surgical VEGF levels were significantly higher than those measured 25-30 days after surgery. Moreover, VEGF concentration differed significantly among cases according to TNM stages and malignant grades $(P<0.0001)$. EGFR mutations and the size of benign pulmonary nodules did not affect the level of serum VEGF significantly.

Conclusion: The serum VEGF levels exhibited relatively high sensitivity and specificity for NSCLC, and may therefore be a useful diagnostic biomarker. Furthermore, the serum VEGF levels could be used to assess prognosis and curative effects.
\end{abstract}

Key words: Non-small cell lung cancer, biomarker, vascular endothelial growth factor, benign pulmonary nodules

\section{Introduction}

Lung cancer remains a leading cause of cancer-related death, with 5-year survival rates of
$<15 \%\left[{ }^{[1]}\right.$. These malignancies can be classified as either non-small cell lung cancer (NSCLC) or small cell lung 
cancer (SCLC), although the former accounts for approximately $85 \%$ of cases ${ }^{[2]}$. Unfortunately, about $24 \%$ of NSCLC are diagnosed at an early stage (local disease, stage I or II), whereas $67 \%$ are diagnosed at more advanced stages (III and IV) ${ }^{[3]}$. Delayed diagnosis of lung cancer leads to increased mortality; patients diagnosed at an early stage (I or II) have a survival rate of $71 \%$, compared with $<2 \%$ for patients with stage IV disease at diagnosis ${ }^{[4]}$.

Asymptomatic patients whose pulmonary nodules were detected incidentally by computed tomography (CT) screening present a challenge to clinicians in terms of determining the characteristics of nodules and providing timely and necessary management. However, screening is associated with increased rates of misdiagnosis, as well as the administration of excessive treatment and wasted medical resources[5]. Therefore, suitable and convenient biomarkers are needed for the differential diagnosis of lung cancer. Although low-dose spiral CT has been recommended as a screening measure for high-risk patients according to the clinical guidelines of the National Comprehensive Cancer Network[6], the clinical value of this modality is limited by high costs and the extremely high false-positive rate $(\sim 94.5 \%)^{[7]}$. Serum biomarkers, such as carcinoembryonic antigen (CEA), cancer antigen (CA)-125, and cytokeratin 19 fragments (Cyfra 21-1), have been used in clinical practice according to the guidelines of the National Academy of Clinical Biochemistry ${ }^{[8]}$. However, these serum markers have low levels of sensitivity (50-60\%), despite high specificities ( $\sim 90 \%)$, and cannot meet the requirements of clinical practice[9][10]. Therefore, more effective and convenient biomarkers remain desirable.

Vascular endothelial growth factor (VEGF), which correlates strongly with abnormal angiogenesis[11], has been shown to play a critical role in lung cancer[12], and previous studies have applied VEGF to differential diagnoses among patients with chronic obstructive pulmonary disease and lung cancer, benign and malignant pleural fluid[13]. Notably, for patients with solitary pulmonary masses detected by chest radiography or CT screening, the levels of VEGF and sVEGFR-1 in bronchoalveolar lavage fluid facilitate the differential diagnosis of primary lung cancer ${ }^{[4]}$. However, to the best of our knowledge, few studies has evaluated the application of serum VEGF to the differential diagnosis of patients with pulmonary nodules from early-stage NSCLC.

A major advance in recent years has been the identification of epithelial growth factor receptor (EGFR) as a driver of disease progression and a therapeutic target in a subgroup of NSCLC patients[15][16]. Approximately $40-50 \%$ of lung adenocarcinomas in Asian populations have EGFR mutations, and less than $10 \%$ of other NSCLC have EGFR mutations ${ }^{[17]}$. It was reported that EGFR mutations were related to the expression of VEGF in tumor tissue[18], but there are no reports on the association between EGFR mutations and level of VEGF in peripheral blood in NSCLC.

In this study, we evaluated whether VEGF could serve as a reliable marker for the differential diagnosis and severity assessment of NSCLC. Accordingly, we compared the serum levels of VEGF, CEA, CA125, and Cyfra 21-1 between patients with NSCLC and those with benign pulmonary nodules (BPN), as well as healthy controls (HC), and evaluated the diagnostic sensitivities and specificities of these serum indexes alone and in combination. Additionally, we analyzed the relationship between VEGF and EGFR mutations/BPN size. Finally, we compared VEGF levels by TNM stage, malignancy grade, and pro- or post-surgical status.

\section{Methods}

\section{Patients and sample collection}

We consecutively enrolled 180 patients with NSCLC, 136 patients with BPN, and 119 HC. Serum samples from all subjects were collected at Sun Yat-Sen University Cancer Center.

Patients with NSCLC were included if they met the following criteria: confirmation of NSCLC via a review of pathologic slides by two independent observers to classify the histologic subtype; no pro-surgical or pro-diagnostic history of antineoplastic therapy, radiotherapy, or chemotherapy; the absence of a second carcinoma, as determined from the clinical history, computed tomography (CT), ultrasonographic examination, and routine laboratory tests; and a post-surgical serum sample (collected from December 2014 to February 2016). Additionally, some patients contributed serum samples both before and within 25-30 days after surgery. The characteristics of the NSCLC patients are presented in Table 1.

Patients with BPN were identified via CT screening and subsequently monitored for 2 years using CT, with no evidence of nodule growth and no clinical diagnosis of cancer. None had a history of previous cancer or chemotherapy. HC were subjects who had not received a diagnosis of malignant or benign tumors after routine examinations, including $\mathrm{CT}$, ultrasonographic examination, and routine laboratory tests. Serum samples were collected from patients with BPN and HC between December 2014 and August 2015. The baseline characteristics of patients with BPN and HC are presented in Table 2. 
Primary tumor samples were obtained from some of the patients. We collected the following clinicopathologic data for each NSCLC patient: age, gender, malignant grade, and pathologic TNM stage. Clinical stage was assessed according to the 7th edition of the Lung Cancer Staging International Division guidelines, which were published by the Union for International Cancer Control and the International Association for the Study of Lung Cancer in 2009. NSCLC was defined based on CT findings and confirmed by histopathology, according to the World Health Organization Classification of Tumors of the Lung. All patients provided written informed consent for their medical information to be stored and used in the hospital database at their first visit to our center. This study was agreed to be conducted by the Institutional Review Board and Human Ethics Committee of Sun Yat-Sen University Cancer Center through the review.

\section{DNA extraction}

DNA was extracted from the $10-\mathrm{mm}$ thick tumor tissue sections microdissected from formalin-fixed paraffin-embedded surgically resected tumor specimens. To obtain genomic DNA, the QiAmp DNA Mini kit (Qiagen, Hilden, Germany) was used according to the manufacturer's instructions.

\section{Scorpions amplification refractory mutation system (ARMS) for the detection of EGFR mutations}

We used the EGFR Scorpions kit (DxS, Manchester, UK), which combines two technologies, ARMS and Scorpions, to detect mutations in real-time polymerase chain reaction (rt-PCR) reactions. All reactions were performed according to the manufacturer's instructions. Real-time PCR was performed with the ABI PRISM 7700 sequence detector (Applied Biosystems, Foster City, CA, USA) under the following conditions: initial denaturation at $95^{\circ} \mathrm{C}$ for 10 minutes, 40 cycles of $95^{\circ} \mathrm{C}$ for 30 seconds, and $61{ }^{\circ} \mathrm{C}$ for 60 seconds with fluorescence reading at the end of each cycle. The data were analyzed with SDS2.0 software (Applied Biosystems) according to the manufacturer's instructions.

\section{DNA sequencing for the detection of KRAS mutations}

DNA amplification was performed using a Techne TC-512 PCR System (Techne, Cambridge, UK). The PCR cycling conditions consisted of an initial denaturation step at $95{ }^{\circ} \mathrm{C}$ for 5 minutes, followed by 35 cycles of $95^{\circ} \mathrm{C}$ for 40 seconds, $56^{\circ} \mathrm{C}$ for 30 seconds, $72^{\circ} \mathrm{C}$ for 30 seconds, and a final extension step at $72{ }^{\circ} \mathrm{C}$ for 6 minutes. The PCR products were separated by electrophoresis in a $2 \%$ agarose gel. The product was excised from the gel and purified with an AxyPrep ${ }^{\mathrm{TM}}$ DNA Gel Extraction Kit (Axygen Biosciences, Union City, CA, USA) following the manufacturer's instructions. The purified PCR products were reamplified by a BigDye Terminator v1.1 Cycle Sequencing Kit (Applied Biosystems) with both primers. The products were sequenced in both directions using the ABI Prism 3700 Analyzer (Applied Biosystems).

\section{Fluorescent in situ hybridization (FISH) analysis of anaplastic lymphoma kinase (ALK) rearrangements}

Bacterial artificial chromosomes, RP11-667I6 and RP11-100C1 (Children's Hospital Oakland Research Institute), were used as break-apart probes for the EML4 and ALK genes, respectively. Bacterial artificial chromosome DNA was labeled with either spectrum red 2-deoxyuridine, 5-triphosphate (dUTP) or spectrum green 11-dUTP by nick translation (Vysis) according to the manufacturer's recommendations. Slides for metaphase FISH from the cell lines were prepared using standard cytogenetic methodologies. The probes were hybridized and washed according to standard FISH procedures. A positive FISH result for $A L K$ rearrangement was defined as $>15 \%$ of tumor cells with a split signal and was confirmed by immunohistochemistry.

\section{Analysis of VEGF levels}

Serum VEGF levels were determined using a double-antibody sandwich enzyme-linked immunosorbent assay (ELISA; WEGO Biology, Weihai, China) according to the manufacturer's instructions. Briefly, $50 \mu \mathrm{l}$ of each serum sample was added per well, followed by $50 \mu \mathrm{l}$ of a horseradish peroxidaseconjugated detection antibody, after which the reaction was incubated for $1 \mathrm{~h}$ at $37^{\circ} \mathrm{C}$. Next, the wells were washed five times with $1 \mathrm{X}$ washing buffer, and incubated with an enhanced chemiluminescent agent in the dark for $3 \mathrm{~min}$ at $18-25{ }^{\circ} \mathrm{C}$. Finally, the chemiluminescence values were detected using a chemiluminescence immunity analyzer (BK-L96C, Zhongshengbaike, Beijing, China).

\section{Analysis of tumor markers}

An Elecsys immunoassay analyzer (COBAS-E602; Roche, Shanghai, China) was used to analyze levels of the tumor markers CEA, CA-125, and Cyfra 21-1.

\section{Specimen characteristics}

All the peripheral blood samples were collected in anticoagulant-free tubes and processed according to standard protocols. Within 30 minutes of collection, the blood samples were centrifuged at 3,000 rpm at 
room temperature for $10 \mathrm{~min}$. Subsequently, the supernatants were divided into $200-\mu 1$ aliquots and stored at $-80^{\circ} \mathrm{C}$ until testing.

Table 1. Level of serum VEGF and clinical characteristics of patients with NSCLC

\begin{tabular}{|c|c|c|c|c|}
\hline Characteristics & Case numbers & Percentage & $\begin{array}{l}\text { VEGF(pg/ml) } \\
\text { Median(range) }\end{array}$ & $P$ Value \\
\hline \multicolumn{5}{|l|}{ Age, years } \\
\hline$<60$ & 77 & 42.78 & $151.2(0.1-880.0)$ & \multirow[t]{2}{*}{0.300} \\
\hline$\geq 60$ & 103 & 57.22 & $136.5(6.1-868.8)$ & \\
\hline \multicolumn{5}{|l|}{ Gender } \\
\hline Male & 103 & 57.22 & $153.6(0.1-880.0)$ & \multirow[t]{2}{*}{0.353} \\
\hline Female & 77 & 42.78 & $128.5(6.1-694.2)$ & \\
\hline \multicolumn{5}{|l|}{ Type } \\
\hline & 155 & 86.11 & $130.8(0.1-868.8)$ & \multirow[t]{6}{*}{0.254} \\
\hline & 11 & 6.11 & 209.3(53.3-694.2) & \\
\hline & 3 & 1.67 & 152.2(139.3-170.7) & \\
\hline & 6 & 3.33 & $227.1(94.8-605.7)$ & \\
\hline & 3 & 1.67 & $150.8(114.9-222.5)$ & \\
\hline & 2 & 1.11 & 287.1(106.5-467.7) & \\
\hline \multicolumn{5}{|l|}{ Grade } \\
\hline 1 & 5 & 2.78 & $50.4(15.6-108.4)$ & \multirow[t]{4}{*}{$<0.0001$} \\
\hline 2 & 65 & 36.11 & $90.2(6.1-313.4)$ & \\
\hline 3 & 53 & 29.44 & $169.5(0.1-694.2)$ & \\
\hline$x$ & 57 & 31.67 & $181.4(0.1-467.7)$ & \\
\hline \multicolumn{5}{|l|}{ T stage } \\
\hline T0 & 3 & 1.67 & $35.7(12.0-81.4)$ & \multirow[t]{6}{*}{$<0.0001$} \\
\hline $\mathrm{T} 1$ & 80 & 44.44 & $100.3(7.2-511.3)$ & \\
\hline $\mathrm{T} 2$ & 62 & 34.44 & $164.2(0.1-868.8)$ & \\
\hline $\mathrm{T} 3$ & 18 & 10.00 & $284.0(163.7-605.7)$ & \\
\hline $\mathrm{T} 4$ & 11 & 6.11 & $162.2(92.4-467.7)$ & \\
\hline $\mathrm{x}$ & 6 & 3.33 & $309.2(159.3-880.0)$ & \\
\hline \multicolumn{5}{|l|}{ N stage } \\
\hline No & 107 & 59.44 & $101.5(0.1-605.7)$ & \multirow[t]{5}{*}{$<0.0001$} \\
\hline N1 & 22 & 12.22 & $140.5(59.8-216.5)$ & \\
\hline N2 & 34 & 18.89 & $228.5(86.1-694.2)$ & \\
\hline N3 & 7 & 3.89 & $301.8(106.5-868.8)$ & \\
\hline$x$ & 10 & 5.56 & $185.9(145.1-880.0)$ & \\
\hline \multicolumn{5}{|l|}{ M stage } \\
\hline M0 & 146 & 81.11 & $119.0(0.1-605.7)$ & \multirow[t]{2}{*}{$<0.0001$} \\
\hline M1 & 34 & 18.89 & 263.1(16.1-880.0) & \\
\hline TNM status & & 0.00 & & $<0.0001$ \\
\hline Stage I & 89 & 49.44 & $86.0(0.1-295.3)$ & \\
\hline Stage II & 25 & 13.89 & $162.8(59.8-605.7)$ & \\
\hline Stage III & 32 & 17.78 & $176.5(53.3-511.3)$ & \\
\hline Stage IV & 34 & 18.89 & 289.3(92.4-880.0) & \\
\hline
\end{tabular}

A Kruskal-Wallis test.

Table 2. The baseline of NSCLC, BPN and HC

\begin{tabular}{lllll}
\hline Characteristics & NSCLC & BPN & HC & $P$ Value \\
\hline Age, years & & & & \\
$<60$ & 77 & 75 & 53 & 0.074 \\
$\geq 60$ & 103 & 61 & 66 & \\
Gender & & & & \\
Male & 103 & 76 & 58 & 0.327 \\
Female & 77 & 60 & 61 & \\
\hline
\end{tabular}

\section{Statistical Analysis}

The statistical analysis was performed using SPSS 20 software (SPSS, Inc., Chicago, IL, USA). The frequency distributions of analytes are presented as means \pm standard errors of the means. Comparisons of VEGF, Cyfra 21-1, CA125, and CEA levels among the three groups were made using the Kruskal-Wallis $(\mathrm{K}-\mathrm{W})$ test with the Bonferroni correction for multiplicity. The Mann-Whitney (M-W) U test was used to analyze differences in VEGF levels according to pathological parameters (e.g., age, gender, $\mathrm{T} / \mathrm{M} / \mathrm{N}$ stage). Differences between pro- and post-surgical VEGF levels were evaluated using the Wilcoxon matched-pairs signed-rank test. Differences in baseline characteristics were assessed using the chi-square test.

To evaluate the diagnostic abilities of the selected biomarkers, area under the receiver operating characteristic (ROC) curve (AUC) values were determined for the indicated patient groups, and the specificity, sensitivity, positive predictive value (PPV), negative predictive value (NPV), and Youden's index were calculated for biomarkers' cut-off levels. Sensitivity was calculated as the true positive rate/(true positive + false negative rate), and specificity was calculated as the true negative rate/(true negative + false positive rate). PPV was calculated as the true positive rate/(true positive + false positive rate), and NPV was calculated as the true negative rate/(true negative + false negative rate). Youden's index values were calculated as the (sensitivity + specificity) -100 , and the highest values were set as the cut-off values. Differences were considered significant at a $P$ value $<0.05$.

\section{Results}

We recruited a total of 435 participants (NSCLC, 180; BPN, 136; HC, 119) for whom the characteristics are summarized in Tables 1 and 2. The VEGF levels of each subgroup and the corresponding $P$ values are listed in Table 1.

\section{VEGF serum levels in patients with NSCLC or BPN and healthy controls}

In an ELISA analysis of the serum VEGF levels of each participant, we determined a median serum VEGF level of $146.23 \pm 95.11 \mathrm{pg} / \mathrm{ml}$ among patients with NSCLC, which was significantly higher than the levels observed in patients with BPN (41.15 \pm 24.56 $\mathrm{pg} / \mathrm{ml}, P<0.0001, \mathrm{M}-\mathrm{W}$ test, Fig. 1a) and HC (35.90 \pm $23.49 \mathrm{pg} / \mathrm{ml}, P<0.0001, \mathrm{M}-\mathrm{W}$ test, Fig. 1a). We additionally analyzed the association between the serum VEGF levels and the clinical characteristics of patients with NSCLC, and found that patients with stage T3-T4 disease had a significantly higher median serum VEGF level $(212.2 \pm 104.3 \mathrm{pg} / \mathrm{ml})$, compared to patients with stage T0-T2 disease (126.3 \pm 114.8 $\mathrm{pg} / \mathrm{ml} ; P<0.0001$, Table 1$)$. In addition, we observed associations between serum VEGF levels and malignancy grade, $\mathrm{N}$ stage, $\mathrm{M}$ stage, and TNM stage (Table 1, Fig. 2). However, we did not observe obvious relationships between serum VEGF levels and other patient characteristics, such as age, gender, or type of cancer. 
A

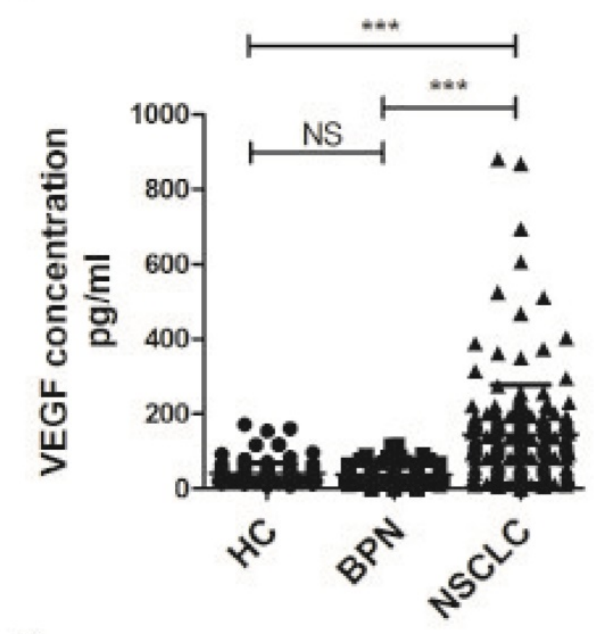

C

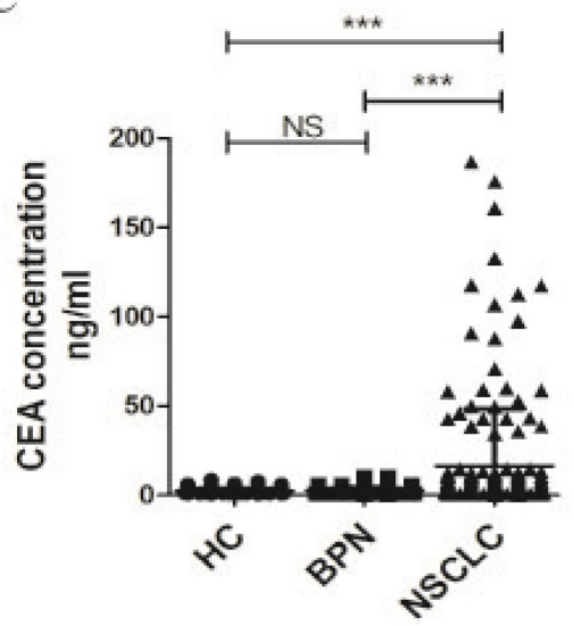

B

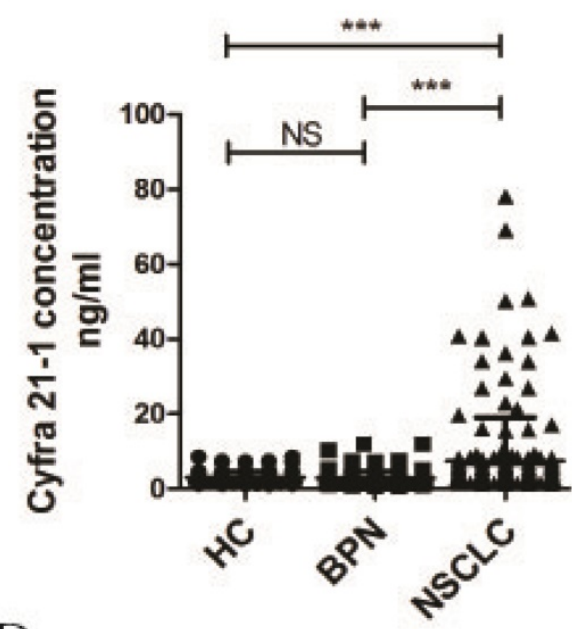

$\mathrm{D}$

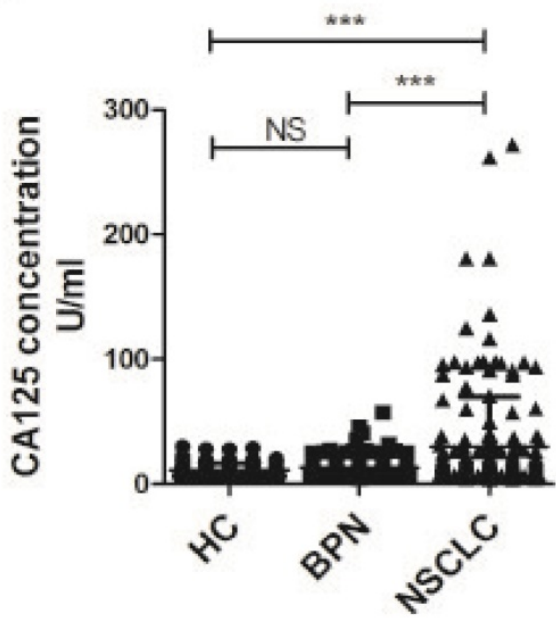

Figure 1. Elevated serum levels in serum samples from patients with NSCLC and controls. Serum levels of VEGF (A), Cyfra 21-1 (B), CEA (C), and CA-125 (D) in HC $(n=119)$, patients with BPN $(n=136)$, and NSCLC $(n=180)$.*** $P<0.0001$. NS, nonsense; HC, healthy controls; BPN, benign pulmonary nodules; NSCLC, non-small cell lung cancer; VEGF, vascular endothelial growth factor; Cyfra 21-1, cytokeratin 19 fragments; CEA, carcinoembryonic antigen; CA-125, cancer antigen 125

\section{ROC analyses of the diagnostic efficacies of VEGF, CEA, CA125, and Cyfra 21-1 and the construction of diagnostic models for NSCLC}

Next, we plotted ROC curves based on the ELISA results to determine the diagnostic effectiveness of the serum VEGF levels for distinguishing NSCLC from BPN and HC, and included the existing clinical biomarkers, CEA, CA125, and Cyfra 21-1, for comparison (Fig. 3 A, B, Table 3). The levels of the individual markers and their predictive values and likelihood ratios in the diagnosis of NSCLC are summarized in Table 3. We used a binary logistic regression to investigate whether diagnostic accuracy could be improved by combining the markers. Notably, the combination of VEGF, CEA, CA125, and Cyfra 21-1 improved the capacity for discrimination and yielded a better optimal diagnostic efficacy for patients with NSCLC [AUC: NSCLC vs. HC $=0.921(0.890-0.952)$ and NSCLC vs. $B P N=0.916$ (0.882-0.949), Table 3], compared with VEGF alone.

Because early diagnosis can significantly improve the 5-year overall survival, we analyzed the role of VEGF in early diagnosis. VEGF had the best diagnostic efficacy among the four biomarkers [AUC: NSCLC vs. HC $=0.747(0.641-0.781)$ and NSCLC vs. $\mathrm{BPN}=0.765$ (0.697-0.833), Fig. 4 A, B, Table 5], and the combination of VEGF, CEA, CA125, and Cyfra 21-1 improved the capacity for discrimination and yielded a better optimal diagnostic efficacy for patients with NSCLC [AUC: NSCLC vs. HC $=0.872(0.822-0.923)$ and NSCLC vs. BPN $=0.869(0.818-0.921)$, Fig. 4 A, B, Table 5], compared with VEGF alone. 
A

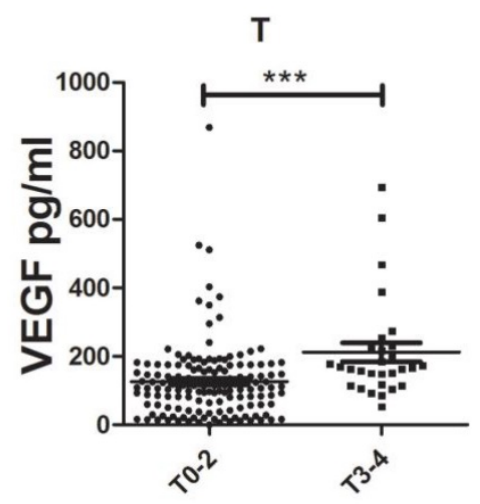

D

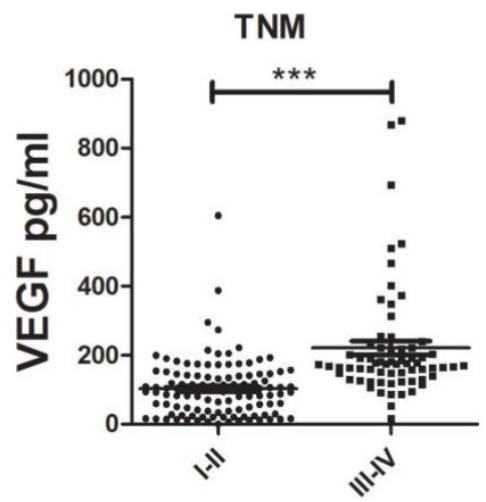

B

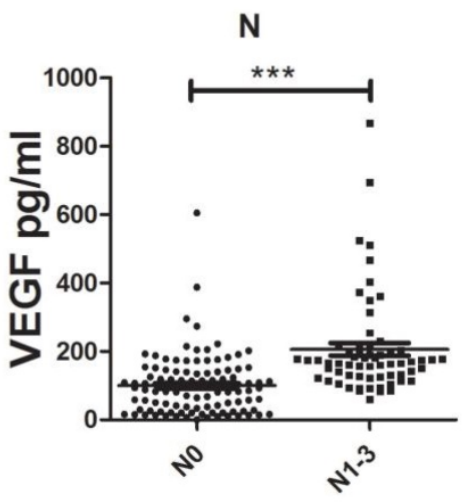

E

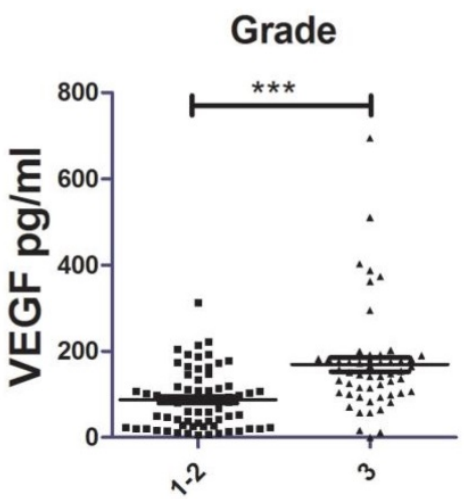

C

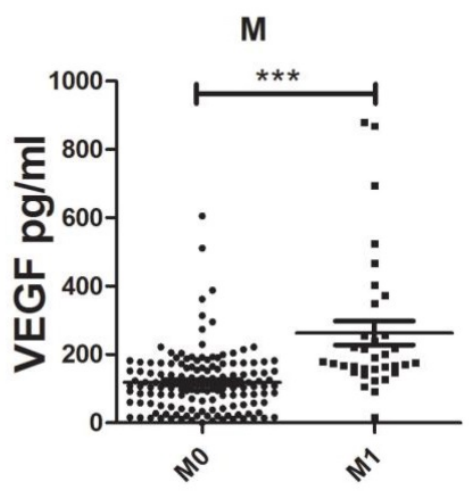

F

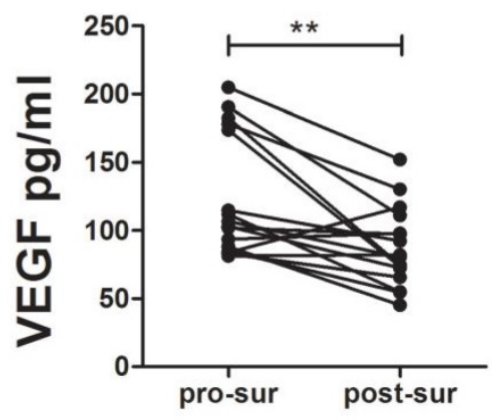

Figure 2. Serum VEGF levels are associated with $\mathrm{T}, \mathbf{N}, \mathbf{M}$ and TNM stages, malignant grades, and surgery. Comparisons of VEGF levels in T0-2 ( $\mathrm{n}=$ $145)$ vs. T3-4 (n=29) (A), N0 $(n=107)$ vs. NI-3 $(n=63)(B), M 0(n=146)$ vs. MI $(n=34)(C)$, and TNM stage I-II ( $n=114)$ vs. III-IV ( $n=66)(D)$ cases, malignant grades $1-2(n=70)$ vs. $3(n=53)(E)$, and pro- vs. post-surgical serum samples $(n=16)$. $* * P<0.01$. VEGF, vascular endothelial growth factor; T, tumor; N, lymph node involvement; $M$ distant metastasis; $G$, malignancy grade; pro-sur, pro-surgical serum; post-sur, post-surgical serum

\section{The size of benign pulmonary nodules did not affect the level of serum VEGF significantly}

We divided patients with BPN into two groups based on the size of the nodules: group 1 length*width $\leq 25 \mathrm{~mm}^{2}$ and group $2>25 \mathrm{~mm}^{2}$. Then, we compared the level of VEGF of the two groups (Fig. 5). The difference in VEGF level between the groups was not significant $(P=0.860)$.

\section{EGFR mutations did not affect the level of serum VEGF significantly}

As KRAS and ALK are downstream of EGFR, we excluded all the case with KRAS mutations or ALK rearrangements. There was no significant difference in VEGF levels between the EGFR mutations group and the wild-type group $(P=0.187$, Fig. 6 A). Mutations associated with enhanced sensitivity to EGFR TKIs are found in exons 18-21 of the TK

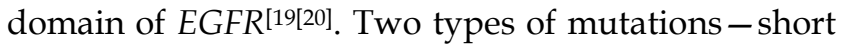
in-frame deletions in exon 19, clustered around the amino-acid residues 747-750, and a specific exon 21 point mutation (L858R)-have been reported to comprise up to $90 \%$ of all activating EGFR mutations ${ }^{[21]}$. Therefore, we divided the cases into two groups: exon 19 mutations and exon 21 mutations. Next, we compared these groups with the EGFR wide-type group (Fig. $6 \mathrm{~B}$ ). There were no significant differences in VEGF level among the groups. The $P$ value between the exon 21 mutation and the wide-type group was 0.091 .

\section{Elevated serum VEGF levels before surgery, compared with after surgery}

We collected both pro- and post-surgical (25-30 days after surgery) serum samples from 16 patients with NSCLC. The post-surgical samples had significantly lower VEGF levels compared to the pro-surgical samples $(124.0 \pm 87.5$ vs. $87.7 \pm 30.5$ $\mathrm{pg} / \mathrm{ml}, P=0.0041$, Fig. $2 \mathrm{~F}$ ).

\section{Discussion}

The distinction of malignant lung tumors from $\mathrm{BPN}$ is a basic problem in the field of respiratory disease. Although imaging techniques such as CT have facilitated the detection of early-stage lung cancers, such analyses are expensive and yield excessively high false positive rates $(>95 \%)^{[22]}$. Other 
diagnostic techniques, such as bronchial microscopy (e.g., bronchoalveolar lavage, biopsy, and needle biopsy) are available, but these are invasive and risky, yield only moderate sensitivity (65-79\%), and have a small scope of application[23]. By contrast, peripheral blood collection is noninvasive, and could be applied to the differential diagnosis of BPN and malignant lung tumors. To date, many serological tumor markers have been used to diagnose cancer, including prostate-specific antigen for prostate cancer, and alpha-fetoprotein for liver cancer[24[25]. However, the currently available clinical biomarkers of lung cancer, Cyfra 21-1, CEA, and CA125 do not have optimal sensitivity and specificity. Accordingly, biomarkers with better diagnostic efficiencies are urgently needed.
A
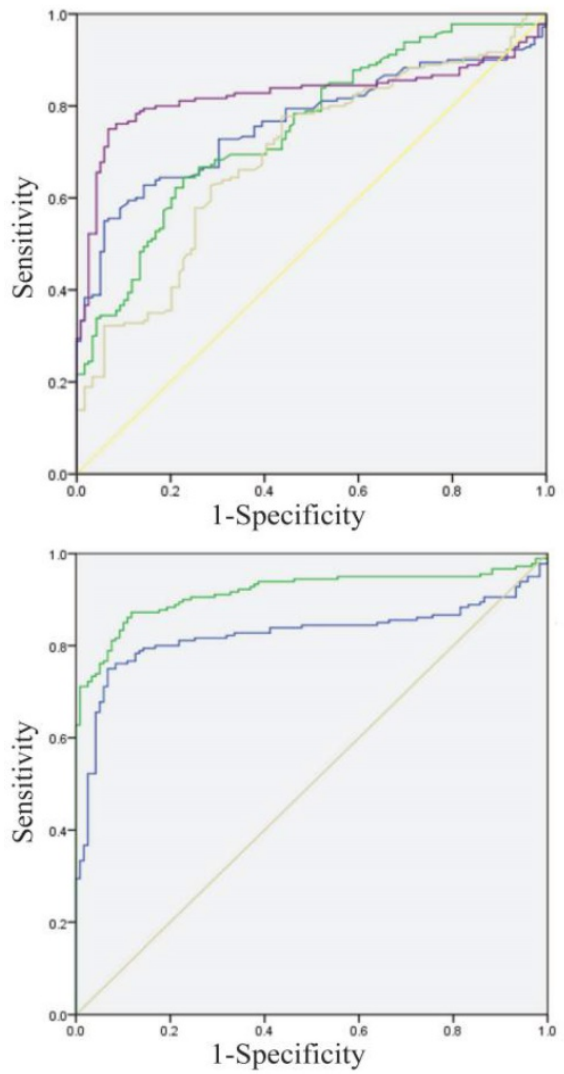

B
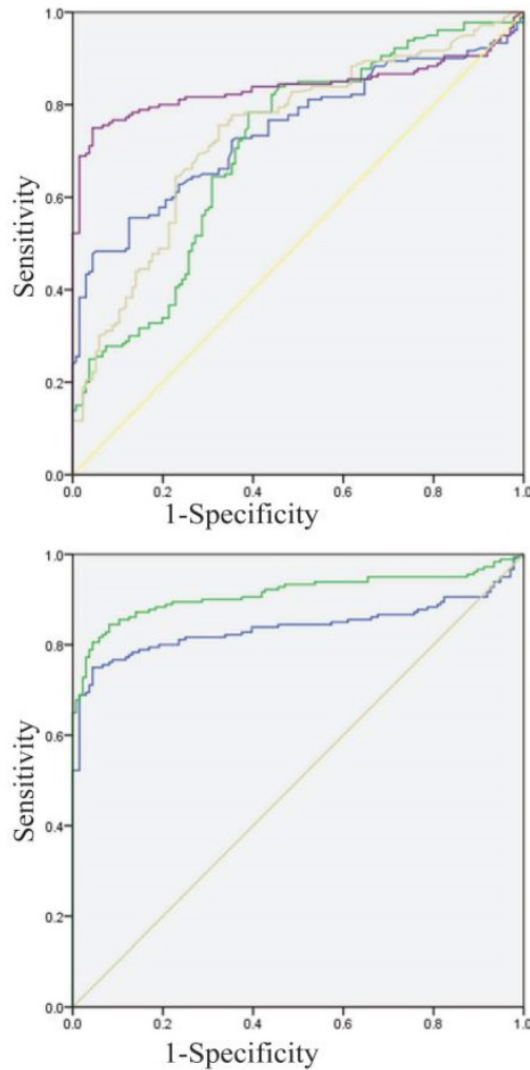
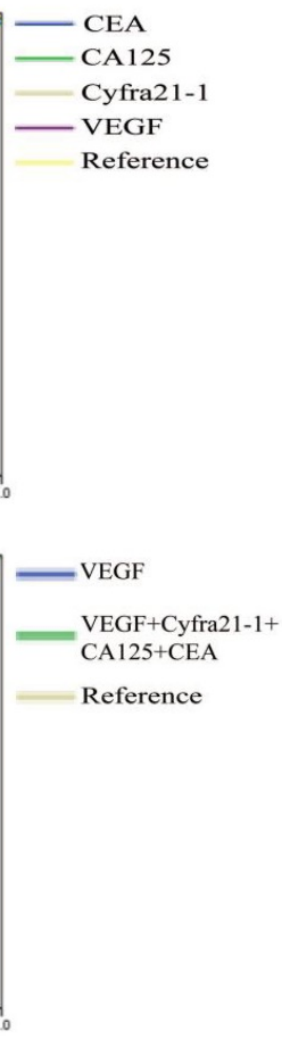

Figure 3. Ability of a combination of VEGF, CEA, CA-125, and Cyfra 21-1 to distinguish between NSCLC and HC/BPN. Comparison of ROC curves of VEGF, CEA, CA-125, Cyfra 21-1, and a combination thereof between NSCLC cases and HC (A) and between NSCLC and BPN (B). ROC, receiver operating characteristic; VEGF, vascular endothelial growth factor; Cyfra 21-1, cytokeratin 19 fragments; CEA, carcinoembryonic antigen; CA-125, cancer antigen 125; NSCLC, non-small cell lung cancer; BPN, benign pulmonary nodules; HC, healthy controls

Table 3. The diagnostic efficiency of models in differentiating NSCLC cases from BPN/HC

\begin{tabular}{|c|c|c|c|c|c|c|c|c|}
\hline & AUC (95\% CI) & Cut-off value & $\mathrm{SN}(\%)$ & SP $(\%)$ & PPV (\%) & NPV $(\%)$ & Positive LR & Negative LR \\
\hline \multicolumn{9}{|l|}{ NSCLC vs. control } \\
\hline VEGF & $0.824(0.773-0.874)$ & 79.3 & 75.0 & 93.3 & 94.4 & 71.6 & 11.2 & 0.268 \\
\hline Cyfra 21-1 & $0.692(0.632-0.752)$ & 3.34 & 62.8 & 71.4 & 76.2 & 54.5 & 2.20 & 0.521 \\
\hline CA125 & $0.752(0.697-0.806)$ & 13.7 & 64.4 & 77.3 & 81.0 & 41.4 & 2.84 & 0.460 \\
\hline CEA & $0.763(0.709-0.816)$ & 3.37 & 62.8 & 85.7 & 87.8 & 59.1 & 4.39 & 0.434 \\
\hline VEGF+Cyfra 21-1+CA125+CEA & $0.916(0.882-0.950)$ & 0.519 & 85.0 & 90.0 & 92.7 & 79.9 & 8.43 & 0.167 \\
\hline \multicolumn{9}{|l|}{ NSCLC vs. BPN } \\
\hline VEGF & $0.839(0.791-0.886)$ & 78.7 & 75.0 & 95.6 & 95.8 & 74.7 & 17.0 & 0.262 \\
\hline Cyfra 21-1 & $0.739(0.709-0.801)$ & 2.82 & 75.6 & 67.6 & 75.7 & 68.1 & 2.33 & 0.361 \\
\hline CA125 & $0.708(0.650-0.766)$ & 10.9 & 78.3 & 61.0 & 72.1 & 65.9 & 2.01 & 0.356 \\
\hline CEA & $0.745(0.691-0.798)$ & 4.65 & 55.6 & 87.5 & 85.5 & 59.8 & 4.45 & 0.507 \\
\hline VEGF+Cyfra 21-1+CA125+CEA & $0.913(0.879-0.947)$ & 0.488 & 84.4 & 91.9 & 93.3 & 81.7 & 10.4 & 0.170 \\
\hline
\end{tabular}

Abbreviations: LR, likelihood ratio; NPV, negative predictive value; PPV, positive predictive value. 
Table 4. The baseline of early stage NSCLC, BPN and HC

\begin{tabular}{lcccc}
\hline Characteristics & I/II & BPN & HC & $P$ Value \\
\hline Age, years & & & & \\
$<60$ & 48 & 75 & 53 & 0.085 \\
$\geq 60$ & 66 & 61 & 66 & \\
Gender & & & & \\
Male & 63 & 76 & 58 & 0.464 \\
Female & 51 & 60 & 61 & \\
\hline
\end{tabular}

Tumor growth depends on angiogenesis[26], a process involving the interactions of many angiogenic and inhibitory factors ${ }^{[27]}$. In this context, VEGF specifically promotes endothelial cell proliferation and induces angiogenesis ${ }^{[28]}$, and its biological effects include increasing vascular permeability, promoting endothelial cell mitosis, and affecting the extracellular matrix. VEGF can also induce the expression of plasminogen activator and its inhibitors, as well as tissue factors and matrix collagenase, which are conducive to vascular growth[29]. As Dvorak et al.[30] reported, the abnormal proliferation of tumor cells leads to a hypoxic environment conducive to VEGF production. In turn, VEGF specifically acts on vascular endothelial cells to promote cellular proliferation, migration, and angiogenesis. In summary, VEGF plays an important role in vascular growth.
A
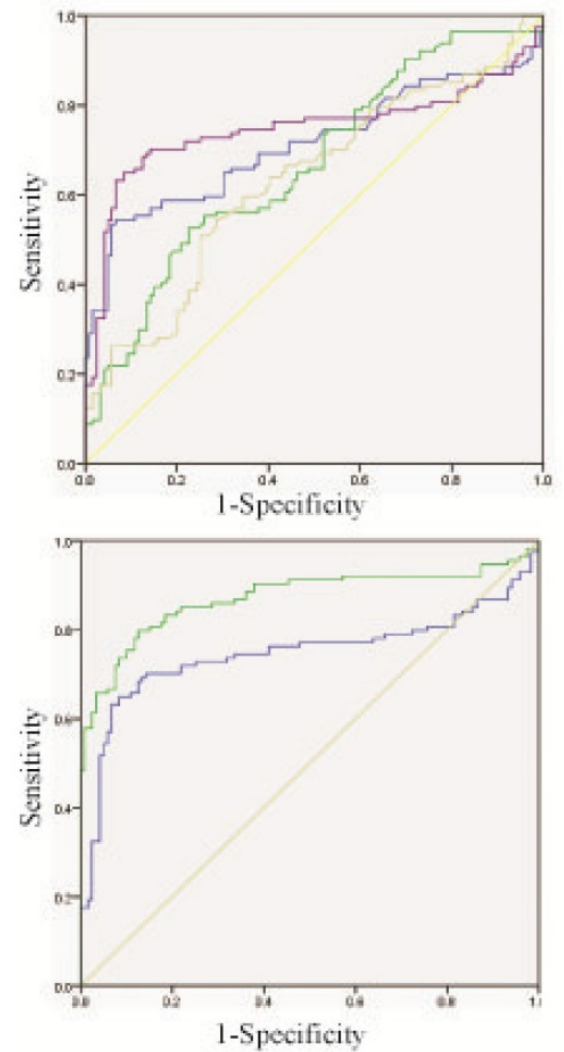

B BPN VS NSCLC
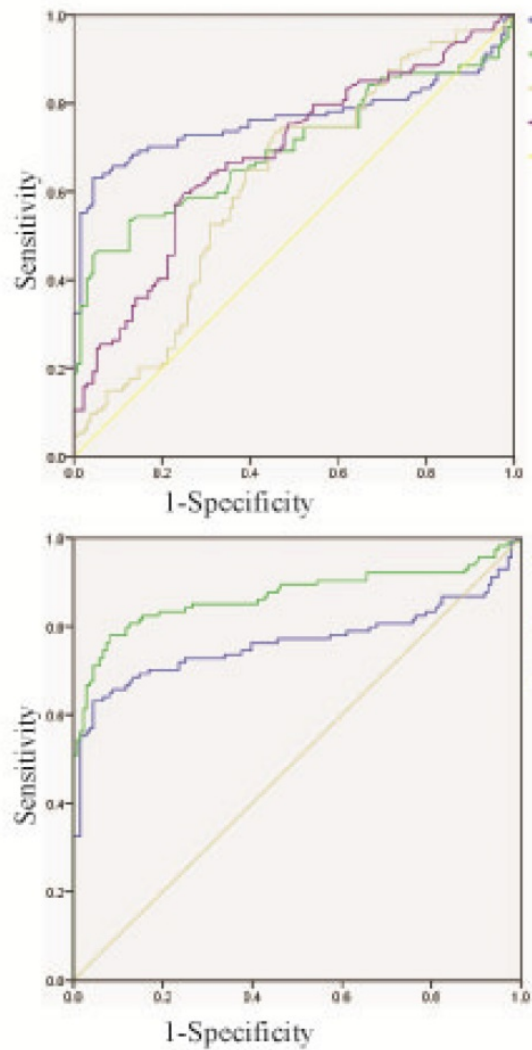

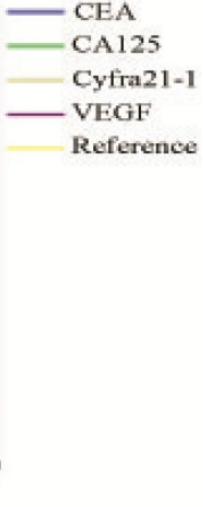

(T)

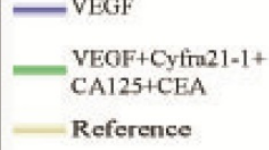

Figure 4. Ability of a combination of VEGF, CEA, CA-125, and Cyfra 21-1 to distinguish between early stage NSCLC and HC/BPN. Early stage NSCLC including stage I and II.

Table 5. The diagnostic efficiency of models in differentiating early stage NSCLC from BPN/HC

\begin{tabular}{|c|c|c|c|c|c|c|c|c|}
\hline & $\operatorname{AUC}(95 \% \mathrm{CI})$ & Cut-off value & $\mathrm{SN}(\%)$ & $\mathrm{SP}(\%)$ & PPV (\%) & NPV $(\%)$ & Positive LR & Negative LR \\
\hline \multicolumn{9}{|l|}{ NSCLC vs. control } \\
\hline VEGF & $0.747(0.641-0.781)$ & 67.3 & 64.9 & 91.6 & 88.5 & 72.3 & 7.72 & 0.383 \\
\hline Cyfra 21-1 & $0.633(0.561-0.704)$ & 3.34 & 54.4 & 71.4 & 65.6 & 61.0 & 1.90 & 0.639 \\
\hline CA125 & $0.667(0.597-0.736)$ & 13.7 & 52.6 & 77.3 & 69.9 & 62.0 & 2.32 & 0.613 \\
\hline CEA & $0.711(0.641-0.781)$ & 4.48 & 54.4 & 93.3 & 89.0 & 67.2 & 8.09 & 0.489 \\
\hline VEGF+Cyfra 21-1+CA125+CEA & $0.872(0.822-0.923)$ & 0.448 & 79.8 & 87.4 & 86.4 & 81.2 & 6.33 & 0.231 \\
\hline \multicolumn{9}{|l|}{ NSCLC vs. BPN } \\
\hline VEGF & $0.765(0.697-0.833)$ & 78.7 & 63.2 & 95.6 & 93.5 & 72.2 & 14.3 & 0.385 \\
\hline Cyfra 21-1 & $0.684(0.617-0.768)$ & 3.22 & 59.6 & 75.0 & 70.5 & 65.0 & 2.39 & 0.538 \\
\hline CA125 & $0.620(0.551-0.690)$ & 10.2 & 73.7 & 54.4 & 61.8 & 67.4 & 1.62 & 0.484 \\
\hline CEA & $0.699(0.630-0.768)$ & 5.50 & 46.5 & 94.9 & 90.0 & 63.9 & 9.03 & 0.564 \\
\hline VEGF+Cyfra 21-1+CA125+CEA & $0.869(0.818-0.921)$ & 0.457 & 78.1 & 91.9 & 90.6 & 80.7 & 9.65 & 0.239 \\
\hline
\end{tabular}

Abbreviations: LR, likelihood ratio; NPV, negative predictive value; PPV, positive predictive value. 


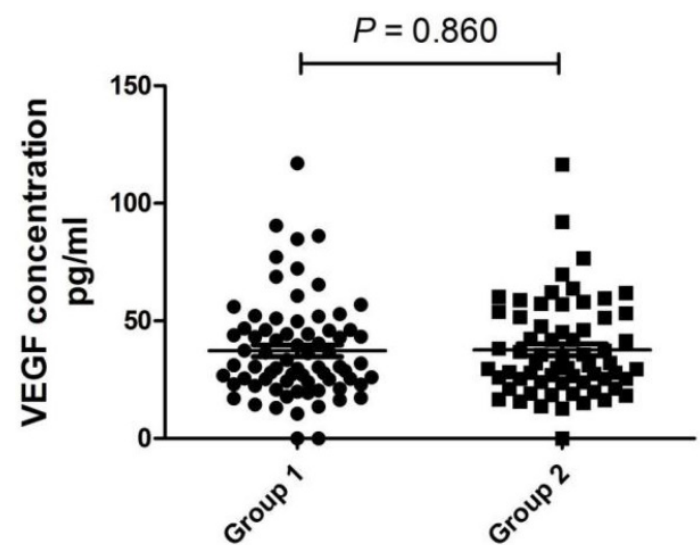

Figure 5. The size of benign pulmonary nodules did not affect the level of serum VEGF significantly. Groupl length*width $\leq 25 \mathrm{~mm}^{2}$ and Group $2>25 \mathrm{~mm}^{2}$.

Table 6. The baseline of BPN

\begin{tabular}{llll}
\hline & Group 1 & Group 2 & $P$ Value \\
\hline Age, years & 38 & 36 & 0.685 \\
$<60$ & 34 & 28 & \\
$\geq 60$ & & & \\
Gender & 39 & 37 & 0.669 \\
Male & 33 & 27 & \\
Female & & \\
\hline
\end{tabular}

Tamura et al. ${ }^{[31]}$ reported significant correlations of serum levels of VEGF with tumor levels of VEGF and microvessel density in patients with NSCLC. Although VEGF is also present in normal tissues, it is only expressed at very low levels under very specific circumstances. Accordingly, serum VEGF levels are likely somewhat reflective of tumor angiogenesis, leading us to our hypothesis that this biomarker could be used to distinguish benign from malignant lung nodules. Serum VEGF levels were also reported to be useful in the diagnosis of various other cancers, including breast, stomach, and colorectal cancer ${ }^{[32]}$. In
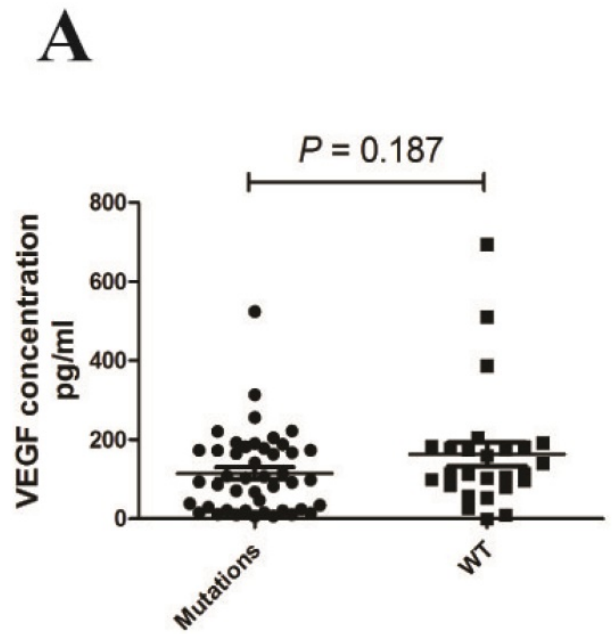

addition, SG Liu et al. [33]detected serum VEGF levels in healthy individuals and patients with benign pulmonary diseases and NSCLC; here, only 37(46.3\%) NSCLC was stage I/II, and the diagnostic effect of VEGF in early-stage NSCLC was not shown. Fifteen of the 40 cases of benign lung disease were inflammatory pseudotumors, and the remainder comprised 14 acute exacerbations of chronic bronchitis, 8 acute attacks of bronchitis asthma, and 3 pulmonary interstitial fibrosis cases. The most difficult problem was identifying early-stage, rather than advanced-stage, NSCLC from BPN. In our study, VEGF performed well in distinguishing early-stage NSCLC from BPN and HC. As reported ${ }^{[34]}$, most cases of BPN are inflammatory pseudotumors, followed by carcinoid and pulmonary tuberculosis. Therefore, we collected additional samples from patients with BPN to further prove the differential diagnostic efficiency of serum VEGF for benign and malignant nodules.

EGFR mutations are closely related to the generation and treatment of lung adenocarcinoma. It has been reported that there is a correlation between the expression of VEGF and EGFR mutations[35]. However, these reports examined VEGF at transcription and protein levels in tumor tissues and did not address the association between EGFR mutation and peripheral blood VEGF. Based on our current results, EGFR mutations are not significantly associated with serum VEGF levels.

Our findings indicate that for NSCLC diagnosis, VEGF was more effective than known clinical serological markers. However, the addition of these markers to VEGF led to a model with an increased diagnostic efficacy. Additionally, we found that the VEGF levels were associated with TNM stages and tumor malignancy grade, independent of age, gender, and type of cancer. We further observed a significant

B

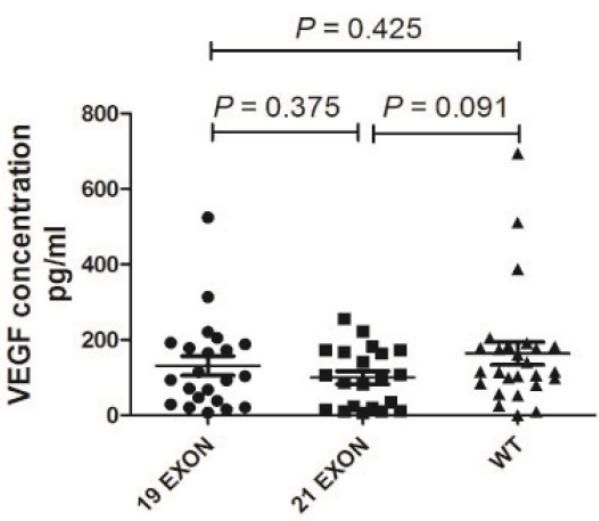

Figure 6. EGFR mutations did not affect the level of serum VEGF significantly. 
decrease in serum VEGF levels from pro- to post-surgery. Taken together, our findings suggest that the serum VEGF levels could be used as a diagnostic biomarker, as well as an indicator of lung cancer prognosis and treatment efficacy.

In summary, our findings suggest that the serum VEGF levels could facilitate the differential diagnosis of NSCLC from BPN. Furthermore, the serum VEGF levels could be used to assess both prognosis and the curative effect.

\section{Acknowledgements}

This study was supported by grants from the National Natural Science Foundation of China (81271902 and 81472008).

\section{Competing Interests}

The authors have declared that no competing interest exists.

\section{References}

[1] Kamangar F, Dores GM, Anderson WF, et al. Patterns of cancer incidence, mortality, and prevalence across five continents: defining priorities to reduce cancer disparities in different geographic regions of the world. J Clin Oncol 2006; 24: 2137-2150.

[2] Siegel RL, Miller KD, Jemal A, et al. Cancer statistics, 2017. CA Cancer J Clin 2017; 67: 7-30

[3] [Internet] Cancer Research UK. Lung cancer incidence statistics. http://www.cancerresearchuk.org/health-professional/cancer-statistics/stat istics-by-cancer-type/lung-cancer/incidence

[4] Zeng Q, Xue N, Dai D, et al. A Nomogram based on Inflammatory Factors C-Reactive Protein and Fibrinogen to Predict the Prognostic Value in Patients with Resected Non-Small Cell Lung Cancer. J Cancer. 2017 Feb 25;8(5):744-753.

[5] Aberle DR, Adams AM, Berg CD, et al. Reduced lung-cancer mortality with low-dose computed tomographic screening. N Engl J Med 2011;365:395-409.

[6] McWilliams A, Tammemagi MC, Mayo JR, et al. Probability of cancer in pulmonary nodules detected on first screening CT. N Engl J Med. 2013;369: 910-919.

[7] Sone S, Li F, Yang ZG, et al. Results of three-year mass screening programme for lung cancer using mobile low-dose spiral computed tomography scanner. Br J Cancer. 2001;84: 25-32.

[8] Berrington de González A, Mahesh M, Kim K-P, et al. Projected cancer risks from computed tomographic scans performed in the United States in 2007. Arch Intern Med. 2009;169: 2071-2077.

[9] Salgia R, Harpole D, Herndon JE II, et al. Role of serum tumor markers CA 125 and CEA in non-small cell lung cancer. Anticancer Res 2001;21:1241-6.

[10] Chi PD, Liu W, Chen H, et al. High-density lipoprotein cholesterol is a favorable prognostic factor and negatively correlated with C-reactive protein level in non-small cell lung carcinoma. PLoS One. 2014 Mar 13;9(3):e91080.

[11] Ahluwalia A, Jones MK, Tarnawski AS, et al. Key role of endothelial importin-a in VEGF expression and gastric angiogenesis: novel insight into aging gastropathy. Am J Physiol Gastrointest Liver Physiol. 2014; 306:G338-45.

[12] Balla MM, Desai S, Purwar P, et al. Differential diagnosis of lung cancer, its metastasis and chronic obstructive pulmonary disease based on serum Vegf, Il-8 and MMP-9. Sci Rep. 2016 Nov 4;6:36065.

[13] Psatha A, Makris D, Kerenidi T, et al. A potential role for VEGF in the diagnostic approach of pleural effusions. J Thorac Dis. 2016 8:1681-7.

[14] Cao C, Sun SF, Lv, et al. Utility of VEGF and sVEGFR-1 in bronchoalveolar lavage fluid for differential diagnosis of primary lung cancer. Asian Pac J Cancer Prev. 2013;14:2443-6.

[15] Reinmuth N, Jauch A, Xu EC, et al. Correlation of EGFR mutations with chromosomal alterations and expression of EGFR, ErbB3 andVEGF in tumor samples of lung adenocarcinoma patients. Lung Cancer. 2008 Nov;62(2):193-201.

[16] Nakade J, Takeuchi S, Nakagawa T, et al. Triple inhibition of EGFR, Met, and VEGF suppresses regrowth of HGF-triggered, erlotinib-resistantlung cancer harboring an EGFR mutation. J Thorac Oncol. 2014 Jun;9(6):775-83.

[17] Suda K, Mitsudomi T. Development of personalized treatments in lung cancer: focusing on the EGFR mutations and beyond. Lung Cancer (Auckl). 2013 Aug 14;4:43-53.
[18] Nakade J, Takeuchi S, Nakagawa T, et al. Triple inhibition of EGFR, Met, and VEGF suppresses regrowth of HGF-triggered, erlotinib-resistant lung cancer harboring an EGFR mutation. J Thorac Oncol. 2014 Jun;9(6):775-83.

[19] Lynch TJ, Bell DW, Sordella R, et al. Activating mutations in the epidermal growth factor receptor underlying responsiveness of non-small-cell lung cancer to gefitinib. N Engl J Med 2004;350:2129-39

[20] Paez JG, Jänne PA, Lee JC, et al. EGFR mutations in lung cancer: correlation with clinical response to gefitinib therapy. Science 2004;304:1497-500

[21] Sharma SV, Bell DW, Settleman J, et al. Epidermal growth factor receptor mutations in lung cancer. Nat Rev Cancer 2007;7:169-81

[22] Aberle DR, Adams AM, Berg CD, et al. National Lung Screening Trial Research Team. Reduced lung-cancer mortality with low-dose computed tomographic screening. N Engl J Med 2011;365:395-409.

[23] Yasufuku K. Early diagnosis of lung cancer. Clin Chest Med. 2010 Mar;31(1):39-47

[24] Izumi K, Ikeda H, Maolake A, et al. The relationship between prostate-specific antigen and TNM classification or Gleason score in prostate cancer patients with low prostate-specific antigen levels. Prostate. 2015 Jul 1;75(10):1034-42.

[25] Liu, Po-Hong, Hsu, Chia-Yang, Hsia, Cheng-Yuan, et al. Prognosis of hepatocellular carcinoma: Assessment of eleven staging systems. J Hepatol. 2016 Mar;64(3):601-8

[26] Folkman J. Angiogenisis in cancer, vascular, rheumatoid and other disease. Nature Med 1995 JAN;1(1):60 27-31.

[27] Carmeliet. P, Jain. RK, et al. Angiogenesis in cancer and other diseases. NATURE. 2000;407: 249-257.

[28] Takahashi $H$, Shibuya $M$, et al. The vascular endothelial growth factor (VEGF)/VEGF receptor system and its role under physiological and pathological conditions. Clin Sci (Lond). 2005;109:227-241

[29] Omiecinski CJ, Aicher L, Holubkov R, et al. Human peripheral lymphocytes asindicatorsofmicrosomal epoxide hydrolase activity in liver and lung. Pharmacogenetics, 1993,3:150-158.

[30] Dvorak HF, Brown LF, Detmar M, et al. Vascular permeability factor/vascular endothelial growth factor, microvascular hyperpermeability, and angiogenesis. Am J Pathol, 1995,146:1029-1039.

[31] TamuraM, Ohta Y, Kajita T, et al. Plasma VEGF concentration can predict the tumor angiogenic capacity in non-small cell lung cancer. Oncol Rep, 2001,8:1097-1102.

[32] Kido-Y. Vascular endothelial growth factor(VEGF)serum concentration changes during chemotherapy in patientswith lung cancer. Kurume Med J, 2001,48:43-47.

[33] Liu SG, Yuan SH, Wu HY, et al. The Clinical Research of Serum VEGF, TGF- $\beta$, and Endostatin in Non-small Cell Lung Cancer. Cell Biochem Biophys. 2015 May;72(1):165-9.

[34] $\mathrm{Hu}$ WD1, Liao MY, Chen JK, et al. Clinical analysis of solitary pulmonary nodule measuring less than $10 \mathrm{~mm}$. Ai Zheng. $2006 \mathrm{Feb}$;25(2):217-9.

[35] Siegelin MD1, Borczuk AC1. Epidermal growth factor receptor mutations in lung adenocarcinoma. Lab Invest. 2014 Feb;94(2):129-37. 